\title{
Understanding of Measuring Accuracy of Liquid Production Chunying Song
}

\author{
No.1 oil Filed Geological Team, No.3 Oil Production plant, Daqing Oilfield Co., Ltd., Daqing \\ 163000, China
}

\begin{abstract}
The liquid production of oil wells is the main basis for the formulation of oil and water well measures and water injection scheme adjustment. The measurement work of oil wells is affected due to the influence of human factors and mechanical equipment, the phenomenon of data admission and actual inconsistency often occur, according to this situation, the problems existing in the process of glass tube oil used in oil fields are comprehensively analyzed, solutions and recommendations are proposed to provide accurate information for the formulation of development plan and dynamic analysis.
\end{abstract}

Keywords: liquid production, measurement, accuracy.

\section{Introduction}

The glass tube measurement oil method has always been the preferred oil measurement method in the oil field because of its advantages of being simple, quick and easy to grasp.

The glass tube measurement oil is a method of constant volume measurement according to the principle of the communication tube. The liquid column in the separator and water column in the glass tube rises to a certain height, because the specific weight of oil-water mixture is different from the water, the rise height is also different. According to the principle of connected pipe balance:

$$
\mathrm{H}_{\text {liquid }} \times \gamma_{\text {liquid }}=\mathrm{h}_{\text {water }} \times \gamma_{\text {water; }}
$$

Obtained from the above formula, $\mathrm{H}_{\text {liquid }}=\frac{h_{\text {water }} \times \gamma_{\text {water }}}{\gamma_{\text {liquid }}}$;

Liquid column weight in the separator $\mathrm{G}=H$ liquid $\times \gamma$ liquid $\times F$

$$
\begin{aligned}
& =\frac{h_{\text {water }} \times \gamma_{\text {water }}}{\gamma_{\text {liquid }}} \times \gamma_{\text {liquid }} \times F \\
& =h_{\text {water }} \times \gamma_{\text {water }} \times F \\
& =h_{\text {water }} \times \gamma_{\text {water }} \times \pi R^{2} ;
\end{aligned}
$$

In the formula, $\mathrm{H}$ liquid, $\mathrm{h}$ water - liquid, water rise height, $\mathrm{m} ; \gamma_{1 \mathrm{iquid}}, \gamma_{\text {water }}$ - Liquid and water specific weight, $\mathrm{t} / \mathrm{m} 3$;

$\mathrm{F}$ — cross-sectional area of separator, $\mathrm{m} 2$;

$\mathrm{R}$ - the inner radius of the separator, $\mathrm{m}$;

If the measured time of the water surface rise height h is t (seconds) when measuring oil, the liquid production per second time

$$
\mathrm{Q}=\frac{\pi R^{2} \times h_{\text {water }} \times \gamma_{\text {water }}}{t} \text {; }
$$

Calculate the formula for daily output (24 hours $=86400$ seconds):

$$
\mathrm{Q}=\frac{\pi R^{2} \times h_{\text {water }} \times \gamma_{\text {water }}}{t} \times 86400 \text {; }
$$

\section{Proposal of Question}

As mining time of oilfield is prolonged, the metering separators are gradually aging, and the dredging maintenance is not timely, at present, after the normal temperature gathering and delivery, the back pressure of several wells rises rapidly, the high casing pressure phenomenon occur now and 
then, it show corrosion and perforation of the pipeline inside the separator, silt blockage, oil well pipeline blockage, low oil level, etc., cause single well oil value to does not reflect the true level, affect the accuracy of data wells and the discovery of problem wells, namely the production dynamics of oil wells cannot be reflected in time. Therefore, it is necessary to improve the measurement accuracy of the liquid production volume of the single well, and then provide accurate data for the dynamic analysis of the oil field.

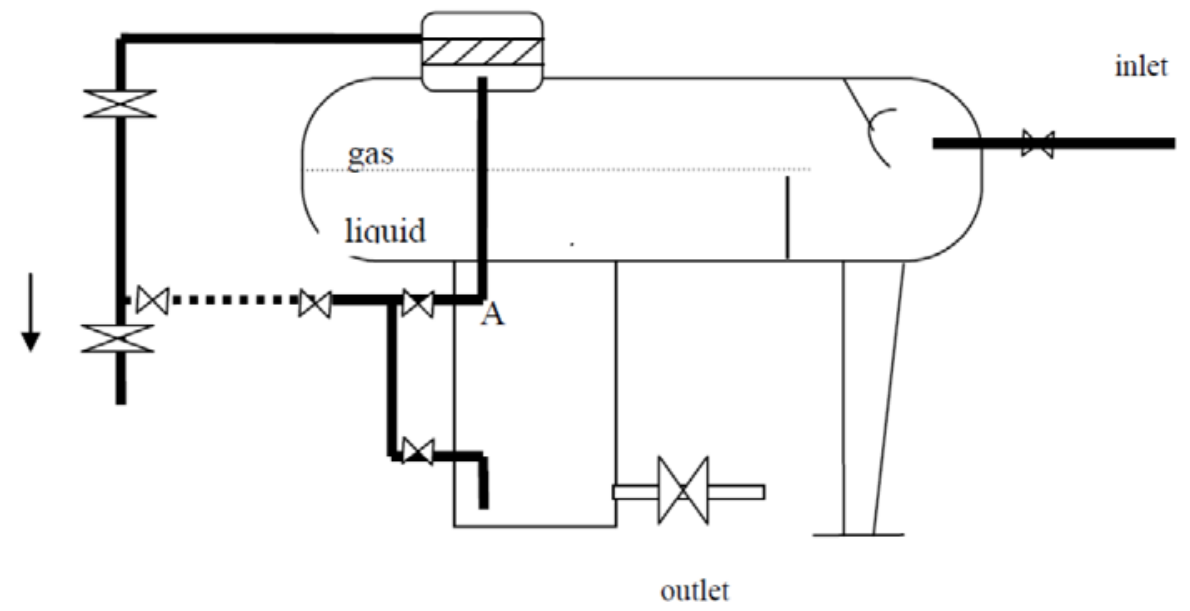

Figure 1. Simple Diagram of Metering Oil Separator

\section{Several Factors Affecting Accuracy of Measuring Oil and Solutions}

\subsection{Separator Problem}

The separator is used for a long time; the long-term oil measurement cause silt and dirt on the bottom of the separator, the separator cannot normally measure the oil, and difficult to enter or drain the liquid, the oil measurement value is less than the actual output, resulting in a negative difference.

By washing the separator, the smoothness of the separator and the accuracy of the oil measurement are guaranteed. We selected the I metering room, which was put into operation in November 2017, and the separator model was $\oint 800$, in early May 2018, we found that all wells output in the metering room decreased, while there is no abnormality for oil casing pressure, current and flow pressure, liquid surface data, when the separator measuring oil, liquid is slow and drainage is normal, on May 15, 2018, we used a professional washing truck to wash the separator. After nearly 3 hours of washing, it was returned to the normal production process, and the filter tank on the well washing truck was emptied, there were many silted fine sediments and sump oil. Before and after washing, the liquid production of all the oil wells in the metering room is measured, after the 11 wells were washed, the daily production liquid recovered 322t, and basically recovered to the normal level before washing, the effect after washing was obvious.

Solution: Regularly clean the separator of metering plant. Each team is required to report the monthly wash separator plan; the separator in the water drive metering plant is washed once every two months, the separator in the metering plant is washed once a month, the separator washing should under the condition that metering plant has a hot well.

\subsection{Effect of the Upper or Lower Tube of the Glass Tube on the Amount of Liquid Production}

Separator measures oil, which is based on the principle of liquid level balance, the method of constant container measurement is used, namely, the liquid level inside the container rises to a certain height, the water surface of the glass tube also rises to the corresponding height, Then use the volumetric formula to calculate the amount of liquid production in one day. However, due to the blockage of the upper and lower flow gates, even though there is already a considerable amount of liquid in the container, the water surface of the glass tube does not rise, this phenomenon is relatively easy to handle, but the number of occurrences is the most. Then, the crude oil in the container penetrates into the glass tube to make the liquid surface blurred, which is not easy to identify. For 
example, the normal daily liquid production of well in No.II metering plant is 89.2t. On April 11, 2018, the oil does not add liquid, the oiler judges that the glass tube is damaged, and returns to the normal level after replacement.

Solution: Washing must be taken before measuring oil. The valve is found badly and replaced in time. At present, venting from the bottom of the glass tube, natural gas is used to wash. It is recommended to alas tube is added to the design standard and install upstream and downstream water mixing gates, seal and wash the glass tube at any time.

\subsection{Effect of Poor Seal of Valve on the Liquid Production of Single Well}

The poor seal of valve of the single well inlet separator results in diversion, so that the oil measurement value is less than the actual production, as shown below figure.

If the valve 1 is not strict, when the B well is measuring oil, the liquid entering the separator will be diverted from valve 1; the accuracy of measuring oil is greatly reduced, the oil measurement value is less than the actual production, and produce negative difference, for example, there is no reason for the decline in the production of a well in the metering plant III, after careful analysis, it was found that the separator valve of this well was poorly sealed, after replacing the valve, the oil was remeasured in this well, and the liquid production increased by $25 t$ before the valve was replaced.

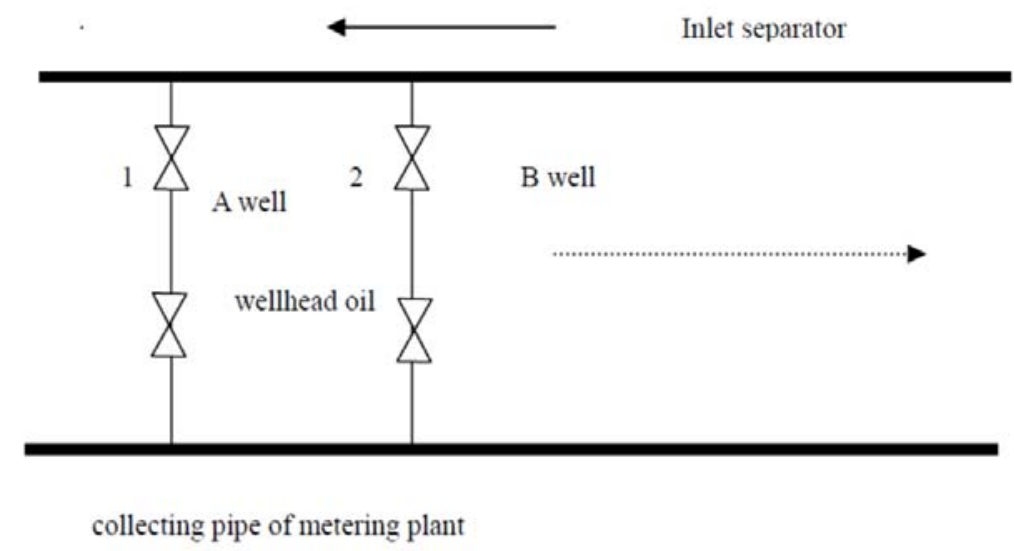

Figure 2. Simple Diagram of Single Well Flow in Metering Plant

Solution: use valves that have a long period of time and are not tight enough are repaired or replaced in time.

\subsection{Effect of Mixing Water}

Before measuring the oil, it must be stopped mix water for 15 minutes to measure the daily production of this well; the specific time for the water mixing should be explored by the metering personnel, if the first measurement time is obviously short, it means that the water mixing time is short.

The oil well with more than 20t liquid production of is measured oil three times, if this well has $30 \mathrm{t}$ liquid production, the diameter of the separator is $800 \mathrm{~mm}$, the height of measuring oil is $50 \mathrm{~cm}$, and the separator constant is 22291.2, The time required for measuring one time is: invert flow, the range from the top to bottom of liquid surface takes at least 3 minutes, the oil measurement is 12 minutes, and the liquid surface is pressed, the total time takes about 20 minutes, therefore, it takes about one hour for measuring the oil for three times, in this way, the wells with low liquid production per day in winter can only be converted, otherwise it will cause the well to be frozen. If the water is not tight, it will also cause the oil measurement value to be inaccurate.

Solution: increase the single well mixing water metering; when the oil well is less than 20t in winter, the oil is mixed with water. Finally, the daily liquid production per well is calculated after deduct the daily water mixing; ensure the accuracy of measuring oil, so as to avoid freezing well. . 


\subsection{High Casing Pressure Affects the Liquid Production of Oil Wells}

The daily working require that the casing pressure of the pumping unit and the screw pump well cannot be higher than 0.6 MPa, and the casing pressure of the electric pump well cannot be higher than 1.0 MPa (except for special wells). After the oil measurement was verified in March 2, 2018, the output increased from 110t to $130.2 \mathrm{t}$, the connected injection well did not raise water, after comprehensive analysis, the reason was that the casing had just finished casing gas, and the casing pressure dropped from 1.1 to $0.53 \mathrm{MPa}$, It can be seen from the above data that the casing pressure of normal oil weel increases by $0.1 \mathrm{MPa}$, and the daily liquid production of single well decreases by $3.2 \%$.

Through the analysis of No. 2 well, it is concluded that the casing pressure oil well is controlled in a reasonable area, and the potential of the oil well can be fully utilized, therefore, the deflation work should be checked regularly and taken as a key work.

\subsection{Effect of Normal Temperature Gathering and Transportation}

In order to comprehensively reduce the energy consumption of the gathering and transportation system, the normal temperature gathering and transportation work will be fully promoted from July 2017. In summer, the daily liquid production is greater than $30 t$, the water content is greater than $85 \%$, or the daily liquid production is $20-30 t$, and the oil well with water content greater than $90 \%$ stop mixing water, the remaining wells mix water $0.5 \mathrm{~m} 3 / \mathrm{h}$ every day; the daily liquid production is greater than 60 t in winter,, and the water content is greater than $85 \%$ and stop mixing water. The daily liquid production is more than 60 t, the water content is less than $85 \%$ or the daily liquid production is 30 $60 \mathrm{t}$, and the oil well whose water content is more than $80 \%$ water is mixed with water $0.5 \mathrm{~m} 3 / \mathrm{h}$. Daily liquid production is $30-60 t$, the oil whose water content is less than $80 \%$ or $10-30$ t daily liquid production is mixed with water $0.8 \mathrm{~m} 3 / \mathrm{h}$. The daily liquid production is less than $10 \mathrm{t}$ and the special well is mixed with water $1 \mathrm{~m} 3 / \mathrm{h}$. The implementation of normal temperature gathering and transportation requires control wellhead back pressure less than 1.0 MPa. Due to the long return line of some oil wells, the back pressure rises faster after stopping the water mixing, resulting in a decrease in the liquid production. For example, after a well stopped mixing water for 25 days, the back pressure is increased from $0.62 \mathrm{MPa}$ to $1.1 \mathrm{MPa}$, and the daily liquid production is reduced from $78 \mathrm{t}$ to $62 \mathrm{t}$, the hot wash pump is started to wash this pipeline, after washing, the daily liquid production liquid returns to normal level, and the back pressure is restored from $1.1 \mathrm{MPa}$ to $0.75 \mathrm{MPa}$. From the above data analysis, the single well back pressure increased by $0.1 \mathrm{MPa}$ and the daily liquid production liquid decreased by $4.4 \%$. Normal temperature gathering and transportation has a certain impact on the daily liquid production of single wells, therefore, it is necessary to timely find the wells with rising back pressure and timely treat, and ensure the accurate measurement of the oil wells.

\subsection{Achieved Results}

Through the above work, the average daily liquid production is from 58944t in September 2017 to 61,729t in May 2018. From the results, improving the accuracy of measuring oil has a certain effect on the governance of the difference, next we continue to strengthen basic management, improve well measurement accuracy and provide accurate basis for dynamic analysis (Figure 3).

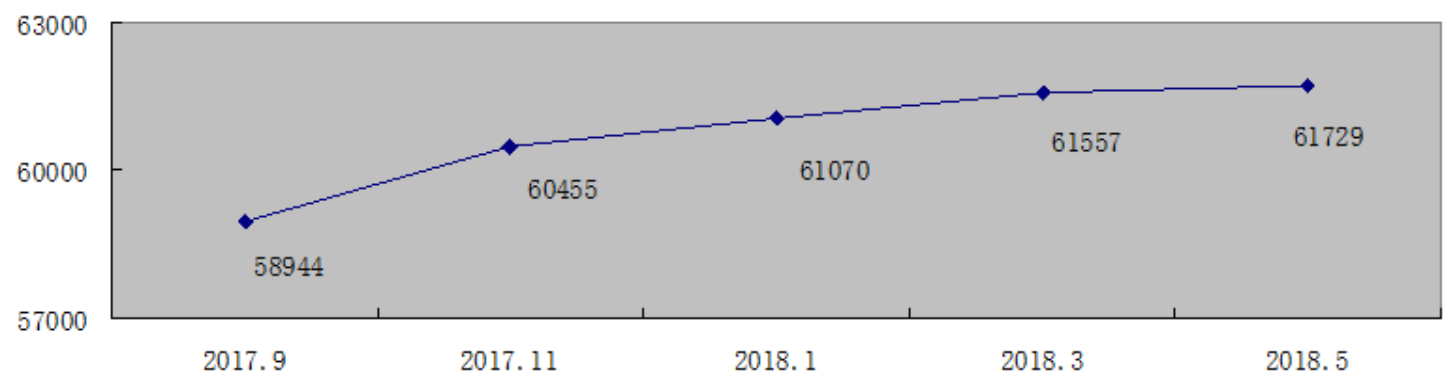

Figure 3. Change Curve of Liquid Production for 2017-2018 


\section{Conclusion}

4.1 Strengthen basic work management, communicate with other functional departments in metering plant management and liquid production measurement, timely discover problems and timely solve, and reduce unnecessary production losses.

4.2 The separator should be cleaned regularly according to the regulations; any poorly sealed valve should be replaced in time; the casing pressure of oil well must be controlled in a reasonable area; the normal temperature gathering and transportation have a certain impact on the daily liquid production of single well, wells with increased back pressure are require timely discovery and timely treatment, ensure accurate measurement of oil wells, meantime it has a good effect on the treatment of the difference.

\section{References}

[1]. Zhang Yingzhi et al. Practice and understanding of oilfield development and adjustment in Sabei Development Zone of Daqing Oilfield, Beijing, Petroleum Industry Press, 2012

[2]. Guan Jinghua, etc., Oil Producer, Beijing, Petroleum Industry Press, 1995 